\title{
Comparative Evaluation of Different Dose of Bupivacaine for Pain Control in Cesarean Section
}

\author{
Anirudh Prasad Mandal', P. K. Gyani ${ }^{2}$, Sandeep Kumar ${ }^{3}$, Rakesh Kumar ${ }^{1}$ \\ ${ }^{1}$ Senior resident, Department of Anesthesia, GMC, Bettiah, ${ }^{2}$ Assistant Professor \& HOD, Department of Anesthesia, GMC, Bettiah, ${ }^{3}$ Junior Resident, \\ Department of Anesthesia, GMC, Bettiah.
}

\section{Abstract}

Background: Spinal anesthesia is the preferred choice of anesthesia for the conduct of emergency and elective cesarean section. Hypotension during spinal anesthesia for caesarean section continues to be a major problem. Aim: The present study investigated the safety and efficacy of $0.25 \%$ hyperbaric bupivacaine on the incidence of visceral pain during cesarean section performed under subarachnoid anesthesia. Subjects and Methods: Sixty patients who were scheduled for elective cesarean section, allocated randomly to one of three groups, according to patient`s height. Group 1, 2 and 3 received 3.2-3.6 mL (8-9 mg), 3.6-4.0 mL (9-10 mg) and 4.0-4.4 mL (10-11 mg) of $0.25 \%$ hyperbaric bupivacaine, respectively. Results: There was no difference in the incidence of visceral pain between three groups ( $p>0.05$ ), but the quality of intraoperative analgesia, as needs for general anesthesia was significantly lower in group 3 than groups 1 and 2 ( $\mathrm{p}<0.05$ ). Conclusion: In conclusion, hyperbaric $0.25 \%$ bupivacaine in the dose of $10-11 \mathrm{mg}$ is safe and effective in obtaining better quality of block in cesarean section, in order of reducing the severity of visceral pain.

Keywords: Spinal Anesthesia, Hyperbaric Bupivacaine, hypotension.

Corresponding Author: Dr. Rakesh Kumar, Senior Resident, Department of Anesthesia, GMC, Bettiah.

Received: July 2019

Accepted: August 2019

\section{Introduction}

Spinal anesthesia is the preferred choice of anesthesia for the conduct of emergency and elective cesarean section in obstetric parturients owing to the associated physiological changes during pregnancy. This include the direct and indirect effects of anesthetics on the fetus and neonates and the benefits and risks of various anesthetic techniques to the mother. The most commonly available methods, to comply the wishes of a parturient, are spinal or epidural anesthesia. Though spinal block is a simple and effective method, because of its reliability and rapid onset of block, it provides precipitous hypotension and difficulty in controlling the level of analgesia limits its use.

The commonly used drugs for epidural anesthesia for cesarean section include $2 \%$ lidocaine, $3 \% \quad 2$ chloroprocaine; $0.5 \%$ bupivacaine and $0.5 \%$ ropivacaine. However, in spite of advantages associated with epidural blocks, there are certain drawbacks also. Despite adequate administration of epidural block, some patients may have inadequate analgesia.

Hypotension during spinal anesthesia for caesarean section continues to be a major problem. Maternal hypotension may be associated with distressing unpleasant symptoms like dizziness, nausea, vomiting. In severe cases, hypotension can result in unconsciousness, apnoea, and cardiac arrest in parturients and may also impair placental perfusion and compromise foetal outcome.

The increasing volume of injected drug as $0.25 \%$ bupivacaine could decrease the incidence or severity of visceral pain (Chung et. al.).

The purpose of this study was to evaluate the safety and efficacy of different volumes of $0.25 \%$ bupivacaine in $5 \%$ glucose on the visceral pain in cesarean section done under spinal anesthesia.

\section{Subjects and Methods}

This prospective study was conducted at Govt Medical College, Bettiah. The study was approved by the institutional research committee. All the participating patients were explained about the procedure and written consent was taken from the participating subjects before the commencement of the procedure. A total of 90 term parturients participated in the study. Class I and II (American society of anesthesiology) parturients were included in the study. Parturients with obstetric complications and fetal compromise were not included in the study.

The participating subjects underwent elective cesarean section under spinal anesthesia. The randomly selected subjects were distributed amongst three groups. The hyperbaric bupivacaine was prepared by adding $0.5 \%$ bupivacaine to $10 \%$ glucose monohydrate. The subjects were distributed in three groups according to height and were administered $3.2-3.6 \mathrm{~mL}, 3.6-4.0 \mathrm{~mL}$, and $4.0-4.4 \mathrm{~mL}$ of the 
drug respectively at L3-L4/ L4-L5. . All subarachnoid blocks were performed by the same anesthetist. The subjects were administered $1000 \mathrm{~mL}$ ringer's lactate solution around 30 mins before and ephedrine 40mg approximately 10 mins before the subarachnoid injection. Standard anesthetic protocol was followed throughout the procedure.

The sensory block was measured using pinprick at $2 \mathrm{mins}$ interval for 10 mins and at 5 mins interval thereafter. The degree of motor block was assessed using modified Bromage scale.

$0=$ no paralysis

$1=$ unable to raise extended leg

$3=$ unable to flex ankle.

The data was collected and was subjected to statistical analysis using SPSS (statistical package for social science) version 10

\section{Results}

There were no statistically significant difference in age, weight, height and gestational age between three groups, but significant difference was in gravidity between group 3 with group 1 and 2. [Figure 1] There was not any significant difference in basic systolic arterial pressure and heart rate, but basic systolic pressure and mean systolic arterial pressure in group 3 were higher significantly than group 2 .

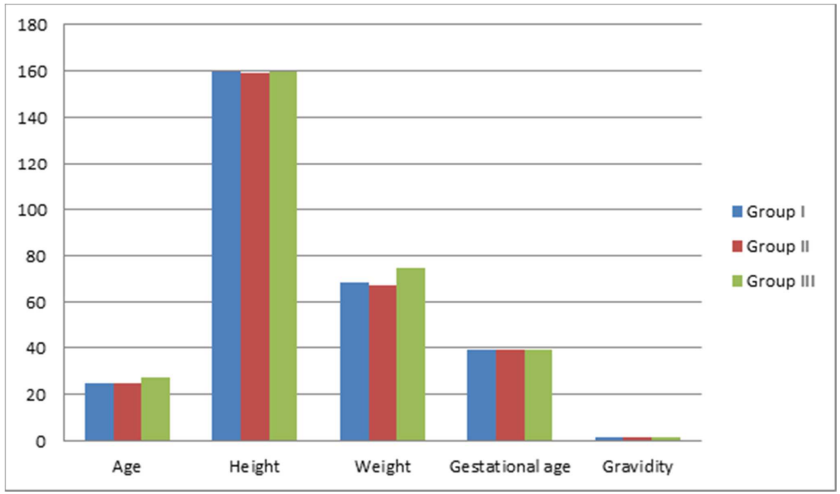

Figure 1: Demographic data

During monitoring of BP in the first 30 min of surgery, the decrease in blood pressure in 6th min post anesthesia was significant in all groups but there was not any difference in hypotension between each group with the other. Maximum changes of systolic arterial pressures, heart rates, needs for ephedrine injection or its dosage didn't change significantly between groups. Comparing of arterial pressures in different min with basic systolic pressure, showed that, there was a significant difference in 4 th $\min$ post anesthesia), this difference is related to the hypotension in group 2 in compared with group 1 . Heart rate at $2 \mathrm{nd}, 4$ th, 6th, 10th and 15 th min after anesthesia had significant difference in compare with basic HR between groups. There were significant differences in mean sensory block level in min 20,25 and 30 between groups but not between two groups with each other. Also, the difference in peak sensory block level was not significant between groups. Mean Motor block in 10th min post anesthesia, showed significant difference between groups with each other. However it was noted that, despite the correlation of block severity in all three groups, after $10 \mathrm{~min}$, the degree of stabilization was very high in group 3, such that all patients in this group had grade 3 of motor block. There was not any difference in onset time of sensory or motor block and the time from onset to T6 level achieved (allowing to surgical incision) between groups, but only in one patient of group 1, the sensory level was T7, such that, she was received additional dose of analgesia (fentanyl+katemine) in 35th $\mathrm{min}$ of anesthesia. None of patients of all groups had pain at incision time, unless, in one case, spinal anesthesia replace with general anesthesia and tracheal intubation, because of the onset of pain.

There were no differences in the time from onset of block to delivery, time from incision to delivery (clamping of umbilical cord), operation time and apgar score of neonates at 5th min, between groups. But 1st min apgar had significant difference between groups. But there was not any difference in every two groups with each other. Of course 1st min apgar was always higher than 7 . [Figure 2]

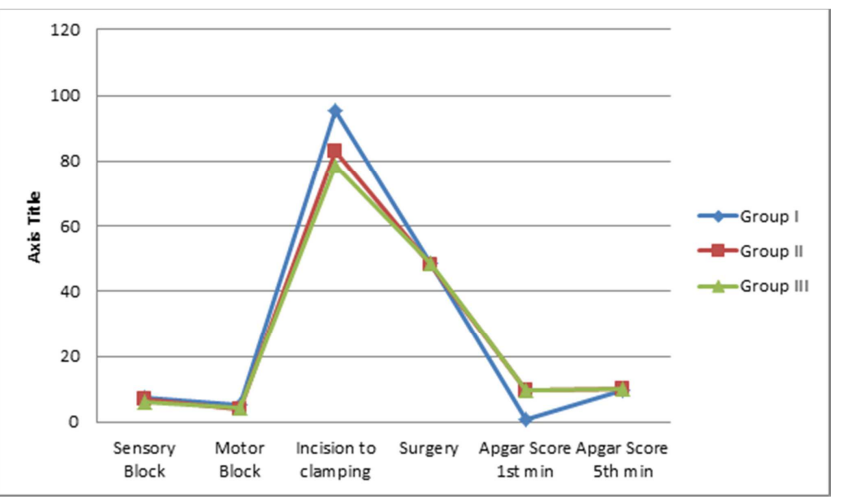

Figure 2: Time Range before achieving peak

Comparing of the quality of anesthesia as the incidence of visceral pain showed no significant difference between groups. There were not any correlation between visceral pain and gravidity, age, weight, peak motor and sensory block level, HR and BP changes, but it was significant correlation between it and the percent of hypertension.

\section{Discussion}

Our study showed that $0.25 \%$ hyperbaric bupivacaine in the range of 10-11 mg can provide satisfactory sensory block and decreases the severity of visceral pain in cesarean section. With this dosage, defect in sensory and motor block can decrease without any rapid and deterious decreasing in arterial blood pressure.

Between demographic variables, although there was not any correlation in gravidity and the incidence of visceral pain, however, it must be considered that gravidity can present mothers culture, experiencing of previous anesthesia, her anxiety and psychological status which can affect the occurrence of visceral pain. Thus, it appears that mother's training programs before and during pregnancy and before anesthesia, can reduce these problems.

Recently a few studies discussed about new properties of bupivacaine which are different from those mentioned earlier. Unlike lidocaine, sympathetic block level is the same 
as sensory block with hyperbaric bupivacaine and as the dose increases, sensory and sympathetic blocks reach a plateau and the quality of block will be better without any increasing in complications (Chung et al., 1996; Echevarria et al., 1996).

In our study the incidence of hypotension was not different between groups $(15.7,30$ and $25 \%$ in group 1, 2 and 3, respectively ).

Pedersen et al. (1989) found the same results, such that the incidence of hypotension in the two group of patients candidated for cesarean section under spinal anesthesia (10$12 \mathrm{mg}$ in group 1 and 7.5-10 mg in group 2 ) was 22-24\%, (Petersen et al., 1989) but Chunget al. (1996) showed the high incidence of hypotension $(40-75 \%)$ in a similar study with the same dosage and volumes of $0.25 \%$ hyperbaric bupivacaine. In our study we elevated parturient's legs 10-15 degree that can probably decrease early hypotension and influence surgical status with relaxing abdominal Rectus muscles. Relative to basic blood pressure the greatest decrease was in 4th min of puncture and unlike lidocaine the onset of hypotension is slow and controllable.

Bradycardia which is a serious complication of spinal anesthesia (Miller et al., 2005) didn't occur in our study, however Chung et al. (1996) reported a $26 \%$ incidence of bradycardia in their study. The reason for this discrepancy is not well known. Although, it has been reported that unlike tetracaine, in subarachnoid injection of hyperbaric bupivacaine, the plasma level of catecholamines elevate and this is probably the reason for the low incidence of bradycardia.

T4 sensory block level usually recommended for cesarean section (Miller et al., 2005), but despite of it, many parturients have several complaints, which are needed for supplementary treatment. In our study, all mothers had at least T6 sensory block level, none of them had pain or discomfort at the incision time, but in one case the spinal anesthesia replace with general anesthesia, because of the onset of pain. Achieving T4 block level, is significantly predictable in group 2 (3.6-4.0 $\mathrm{mL})$ and group $3(4.0-4.4 \mathrm{~mL})$ than group $1(3.2-3.6 \mathrm{~mL})$ and the variance is lower in group 3 than group 2.

Cesarean section is a lower abdomen operation, however, pressures and tractions, applied to the upper part of the abdomen for fetal extraction and, on the other hand, shedding of blood and amniotic fluid to the abdomen cavity, high traction of fallopian tube mesentery at the time of uterine extraction or cleaning of abdomen cavity from blood, all can excite the upper abdomen regions and this problems, need for higher sensory block levels which can be easily accomplished with clinically using doses, but the quality of block will be varied with different doses (Chung et al., 1996). Petersen achieved the same sensory block level with 7.5-10 $\mathrm{mg}$ and $10-12 \mathrm{mg}$ of $0.5 \%$ hyperbaric bupivacaine, but the incidence of visceral pain was significantly low with 10-12 mg (31.6 versus 70.5\%) (Pedersen et al., 1989).

Chung et al. (1996) found the low incidence of visceral pain and the need for supplementary analgesic treatment in group $2(3.6-4.0 \mathrm{~mL})$ and group $3(4.0-4.4 \mathrm{~mL})$ than group 1 (3.2-
$3.6 \mathrm{~mL}$ ). But in our study there was no difference in visceral pain between three groups.

Supplementary analgesia (Inhalational or interval) currently recommended in spinal anesthesia. If the quality of anesthesia is referred to the defect in the severity of block, it was seen that this defect will be decline with increasing the dose of the drug and will be controlled easily with minimal interventions (assurance of mother, intravenous administration of low dose analgesics or sedatives). But in spite of achieving the same sensory block level with high volumes, the defect in the severity of block in low volumes will be more and needs for aggressive supplemental treatment, such that from 6 patients which were received general anesthesia or more than $100 \mu \mathrm{g}$ fentanyl and considered as weak quality of sensory block, 5 patients was from group 1 (3.2-3.6 mL) and one patient was from group 2 (3.6-4.0 mL) and none of patients of group 3 need for this aggressive treatment. It was resulted that better and predictable quality of sensory block will be achieved with increasing volumes of the drug.

\section{Conclusion}

Increasing volumes of $0.25 \%$ hyperbaric bupivacaine may be a safe and effective method for decreasing visceral complaints of mothers undergoing cesarean section with spinal anesthesia.

It was needed for more studies and researches about visceral pain. For this reason, with regard to several physiologic, anatomic, surgical, medical, neurological and psychological aspects, which affect visceral pain and can not be controlled only by anesthesiologist, it appears that a team works with a wide range of responsibility and teaching programs from prepartum to immediately before and after anesthesia is required.

\section{References}

1. Alahuhtas, T., K. Saarela, A.I. Hollmen and H.H. Edstrom, 1990. Visceral pain during cesarean section under spinal and epidural anesthesia with bupivacaine. Acta Anesth. Scand., 34: 95-98.

2. Chestnut, D.H, 2004. Obstetric Anesthesia, Principles and Practice. 3rd Edn., Mosby, New York.

3. Choi, D.H., H.J. Ahn and M.H. Kim, 2000. Bupivacaine: Sparing effect of fentanyl in spinal anesthesia for cesarean delivery. Reg. Anesth. Pain Med., 25: 240-245.

4. Chung, C.J., S.H. Bae, K.Y. Chae and Y.J. Chin, 1996. Spinal anesthesia with $0.25 \%$ hyperbaric bupivacaine for caesarean section: Effects of volume. Br. J. Anesth., 77: 145-149.

5. Echevarria, M., F. Caba, L. Bernal, J.A. Pallares and R. Rodriguez, 1996. Effect of the local anesthetic on visceral pain in cesarean sections done under intradural anesthesia.

6. Rev. Esp. Anestesiol. Reanim., 43: 2-6.Hirabayashi, Y., K. Saitoh, H. Fukud and R. Shimizu, 1995. Visceral pain during caesarean section: Effect of varying dose of spinal amethocaine. Br. J. Anesth., 75: 266268.

7. Miller, R.D., A. Lee-Fleisher and A. John, 2005. Anesthesia. 16th Edn., Churchill Livingston, Philadelphia, pp: 2323-2329, 1661-1677.

8. Pedersen, H., A.C. Santos, E.S. Steinberg, H.M. Schapiro, T.W. Harmon and M. Finster, 1989. Incidence of visceral pain during cesarean section: The effect of varying doses of spinal bupivacaine. Anesth. Analg., 69: 46-49. 


\section{Mandal et al: Different Dase of Bupiracaine far Pain Cantral in Cesarean Section}

0

Copyright: (c) the author(s), publisher. Academia Anesthesiologica International is an Official Publication of "Society for Health Care \& Research Development". It is an open-access article distributed under the terms of the Creative Commons Attribution Non-Commercial License, which permits unrestricted non-commercial use, distribution, and reproduction in any medium, provided the original work is properly cited.

How to cite this article: Mandal AP, Gyani PK, Kumar S, Kumar R. Comparative Evaluation of Different Dose of Bupivacaine for Pain Control in Cesarean Section. Acad. Anesthesiol. Int. 2019;4(2):122-125.

DOI: dx.doi.org/10.21276/aan.2019.4.2.29

Source of Support: Nil, Conflict of Interest: None declared. 